\title{
Number of Spontaneous Abortions
}

National Cancer Institute

\section{Source}

National Cancer Institute. Number of Spontaneous Abortions. NCI Thesaurus. Code C120837.

A measurement of the total number of spontaneous abortions (in which the fetus is less than 20 weeks gestational age) experienced by a female subject. 